\title{
Tyrosine crystals in salivary gland tumours
}

\author{
K THOMAS, MSR HUTT \\ From the Department of Histopathology, St Thomas's Hospital Medical School, London SE1 7EH
}

SUMMARY Tyrosine crystals were seen in 24 of 113 salivary gland pleomorphic adenomas from Malawi and one of six carcinomas arising in a pleomorphic adenoma; they were present in tumours from all common anatomical sites. They were not found in 71 other salivary tumours of varying histological type. Similar crystals were found in one out of 56 pleomorphic adenomas from patients operated on at St Thomas's Hospital. No excess tyrosine was demonstrated in a tumour containing many crystals. Their greater frequency in black patients is confirmed but an adequate explanation for this phenomenon has not been found.

During a study of the histopathology of salivary gland tumours received from patients in Malawi ${ }^{1}$ tyrosine crystals were seen in several pleomorphic salivary adenomas. As there are few reports of crystals in these tumours, we decided to re-examine all the salivary gland tumours to see how often this association occurred and whether it was found in other salivary tumours.

\section{Material and methods}

Since 1968, St Thomas's Hospital Medical School has provided the only histopathological service for the whole of Malawi. All histopathological material from tumours of major and minor salivary glands during the years 1969-77 were re-examined. Where crystalline structures were seen on haematoxylin and eosin-stained sections, Millon's stain was performed to identify them as tyrosine. A search for crystals was also made of pleomorphic adenomas received from patients at St Thomas's Hospital over a six-year period.

\section{Results}

Salivary gland tumours were received from 190 patients in Malawi. There were 113 pleomorphic adenomas and six which showed carcinoma developing in a pleomorphic adenoma. Crystals were seen in 24 pleomorphic adenomas (Fig. 1), and in one carcinoma developing in an adenoma. No crystals were seen in any other type of tumour. One tumour from a caucasian was found to contain crystals among 56 examined from St Thomas's Hospital patients. This was a pleomorphic adenoma from the parotid of a

Accepted for publication 11 February 1981

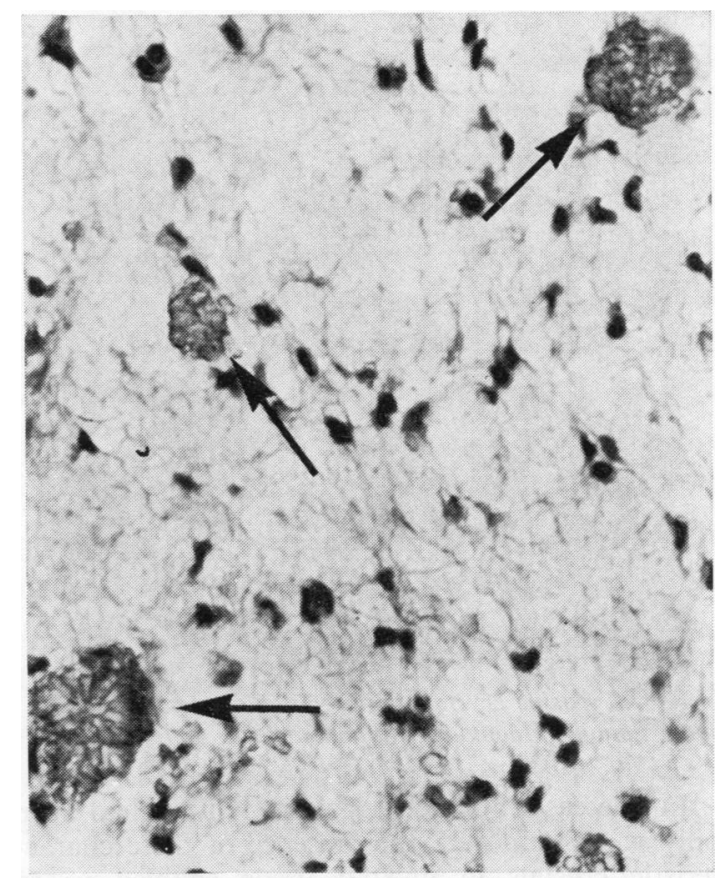

Fig. 1 Groups of tyrosine crystals (arrows) in a myxoid area of a pleomorphic adenoma. Haematoxylin and eosin $\times 400$.

42-year-old man. Of the Malawi patients 13 were male and 12 female. The average age of those with tyrosine crystals was 32.5 yr compared with an average age of $39.5 \mathrm{yr}$ for all those with pleomorphic adenomas. The site of the tumours was as follows: parotid 11; submandibular 4 ; palate 4 ; cheek 5 ; upper lip 1. The site of the tumour was classified as 
cheek where it was not clear from the clinical information and histology whether it had arisen from parotid or buccal glands. The tumours themselves were typical pleomorphic salivary adenomas with both myxochondroid and epithelial areas. However, the tumours in which crystals were found tended to have a predominant myxochondroid component and the crystals were mostly deposited in the myxoid areas (Fig. 1), with overspill to epithelial areas only where deposition was heavy (Fig. 2). In most tumours the crystals were confined to localised areas but the deposition varied from a few crystals in small foci to heavy deposition throughout much of the tumour. The crystals were arranged in the form of rosettes (Fig. 3) usually staining pink with haematoxylin and eosin but were occasionally slightly basophilic. When sections were stained with Millon's stain the crystals were a reddish brown colour.

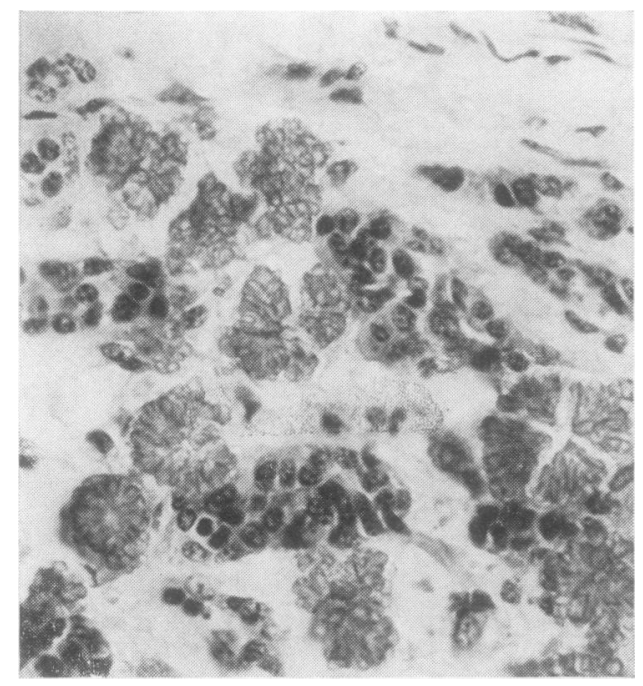

Fig. 2 Tyrosine crystals in an epithelial area of the same tumour. Haematoxylin and eosin $\times 400$.

In one case in which a palatal tumour had been only partly removed, the presence of these characteristic crystals in myxoid tissue enabled us to establish the diagnosis of pleomorphic adenoma.

\section{Discussion}

There are only a few reports of crystalline structures in salivary tumours. Bullock ${ }^{2}$ noticed large numbers of tyrosine crystals in a mixed parotid tumour, and Thackray and Lucas ${ }^{3}$ reported having seen tyrosine crystals in four pleomorphic adenomas from blacks and in one from a white patient. The Millon reaction used to identify tyrosine crystals depends on the

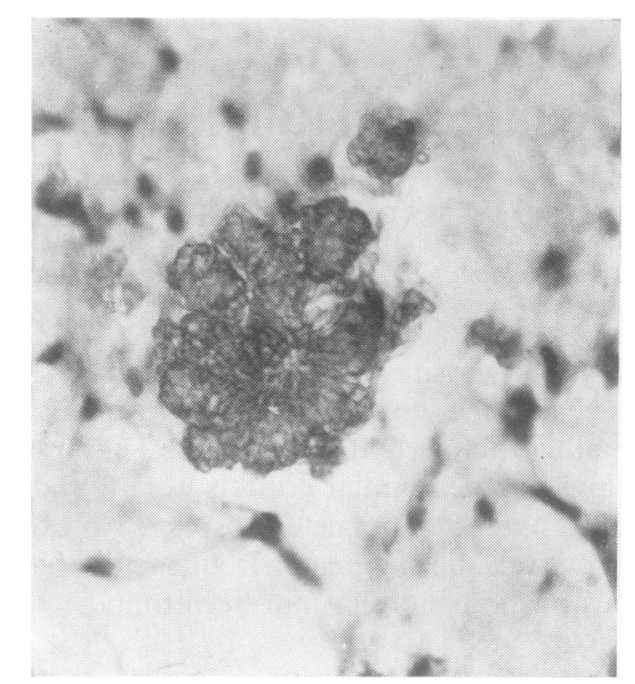

Fig. 3 Radial rosette appearance of tyrosine crystals. Haematoxylin and eosin $\times 1000$.

presence in the protein molecule of the hydroxyphenol group. It is given by any phenolic compound which is unsubstituted in the position meta to the hydroxyl group. As far as mammalian tissues are concerned it is specific for tyrosine. ${ }^{4}$

A suitable explanation for the presence of tyrosine crystals in these tumours is hard to find. There is no correlation with the age of the tumour or of the patient. Our findings confirm the impression that crystals are more common in black races (24 out of 113 pleomorphic adenomas, $21 \%$ ) than in whites ( 1 out of $56,1 \cdot 8 \%$ ). These racial differences suggest that there might be a relation with the involvement of tyrosine in pigment metabolism; however, this offers no explanation for their deposition in a tumour. Experimental administration of excess phenylalanine and tyrosine in rats causes pathological changes in many tissues but no crystal deposition ${ }^{5}$ and tyrosine crystals are not seen in tissues in human tyrosinosis where blood and urine concentrations are high. Amino acids were identified by paper chromatography in portions of homogenised and hydrolysed tissue from a tumour with crystals and a tumour without crystals but the concentrations found including tyrosine were similar in both. The deposition of these crystals, particularly in black patients with pleomorphic adenomas, remains a mystery.

Our thanks are due to the clinicians in Malawi who provided the material for this study, the Medical Laboratory Scientific Officers of this department for technical help and Mr V Clark for assistance with the photomicrography. 


\section{References}

1 Thomas KM, Hutt MSR, Borgstein J. Salivary gland tumors in Malawi. Cancer 1980;46:2328-34.

2 Bullock WK. Mixed tumour of parotid gland with tyrosine crystals in the matrix. Am J Clin Pathol 1953;23:1238-9.

${ }^{3}$ Thackray AC, Lucas RB. Tumours of the major salivary glands. Washington, DC: Armed Forces Institute of Pathology, 1974:32.

4 Pearse AGE. Histochemistry theoretical and applied. J \& A Churchill Ltd, 1972:107, 127.
${ }^{5}$ Klavins JV. Pathology of amino acid excess. Archives of Pathology 1967;84,3:238-50.

Requests for reprints to: Professor MSR Hutt, Department of Histopathology, 2nd Floor, North Wing Block, St Thomas's Hospital Medical School, London SE1 7EH, England. 